\title{
Synthesis and Evaluation of Molecularly Imprinted Polymer for the Determination of the Phthalate Esters in the Bottled Beverages by HPLC
}

\author{
Ya-Feng Jin, ${ }^{1}$ Yi-Jun Zhang, ${ }^{1}$ Yu-Ping Zhang, ${ }^{1}$ Jun Chen, ${ }^{1,2}$ \\ Xiao-Mao Zhou, ${ }^{2}$ and Lian-Yang Bai ${ }^{2,3}$ \\ ${ }^{1}$ Henan Institute of Science and Technology, Xinxiang 453003, China \\ ${ }^{2}$ Pesticide Research Institute, Hunan Agricultural University, Changsha 410128, China \\ ${ }^{3}$ Hunan Institute of Humanities, Science and Technology, Loudi 417000, China \\ Correspondence should be addressed to Yu-Ping Zhang; beijing2008zyp@gmail.com
}

Received 18 October 2012; Accepted 24 February 2013

Academic Editor: Manuela Curcio

Copyright (c) 2013 Ya-Feng Jin et al. This is an open access article distributed under the Creative Commons Attribution License, which permits unrestricted use, distribution, and reproduction in any medium, provided the original work is properly cited.

A molecularly imprinted polymer (MIP) was prepared in acetonitrile by bulk polymerization, using di-n-octylphthalate (DOP) as a template molecular, $\alpha$-methacrylic acid (MAA) as a functional monomer, and ethylene dimethacrylate (EDMA) as a crosslinker. Characterization and evaluation of the prepared MIP were carried out by scanning electron microscope (SEM), infrared absorption spectroscopy (IR), and the Scatchard analysis, respectively. Through the optimization of washing solvent, eluting solvent amount, flow rate of loading solution, and loading sample volume, an analysis method was established for DOP related compounds with high selectivity and sensitivity by using the selective molecularly imprinted solid-phase extraction (MI-SPE) technique. Moreover, under the optimal conditions, the extraction effects were comparatively investigated by using MIP cartridge, NIP cartridge, and the commercial PLS cartridge used especially for phthalic acid esters (PAEs), respectively. The results showed that the recoveries of spiked PAEs are in the range of $90.4 \%-97.8 \%$ with the relative standard deviation (RSD) of $1.6 \%-3.8 \%$ on the resulted MIP cartridge, whilst lower recoveries were obtained ranging from $80.2 \%$ to $88.9 \%$ with an RSD of $1.4 \%-5.2 \%$ on the commercial PLS cartridge.

\section{Introduction}

Phthalate acid esters (PAEs) have a wide variety of industrial, agricultural, and domestic applications, but by far the most important is their use as plasticisers that can increase process ability, flexibility, or extensibility or to decrease hardness or stiffness $[1,2]$. The orthophthalate diesters, for use as plasticisers, are produced by the esterification of phthalic anhydride using alcohols of carbon chain length in the approximate range $4-12$. The physical rather than chemical incorporation of phthalates in the polymeric matrix ensures that they are widespread contaminants. Penetration of phthalates into the ecosystem, food chain or in wastewater effluents occurs during the production phase and via leaching and volatilization from plastic products during their usage and/or after disposal. Current research efforts are directed towards the development of simple and sensitive analytical methods, capable of accurately determining the trace quantities of phthalates in different complex samples [3]. Some preconcentration techniques were generally selected as the sample preparation procedures, based on liquid-liquid extraction, solid-phase extraction (SPE), and solid-phase microextraction (SPME), followed by gas chromatography (GC), liquid chromatography (LC), capillary electrophoresis, or gas chromatography and mass spectrometry (GCMS) [4-10]. As a traditional pretreatment method, SPE is widely used to preconcentrate and clean up the target compound prior to determining the target compounds in complex samples using chromatographic or other analytical methods [11-13]. In these studies, regular commercial SPE 
cartridges were employed. These sorbents retain analytes by nonselective hydrophobic or polar interactions that lead to a partial coextraction of interfering substances. In order to facilitate the trace enrichment of analytes and enhance the selectivity of the extraction and to develop new functionalized polymeric selective sorbents may be a promising way. MIP is synthetic polymers possessing specific cavities designed for a target molecule, which exhibit many outstanding advantages such as high selectivity and physiochemical stability and afford specific recognition against the imprinted molecules and structurally related compounds $[14,15]$. Therefore, the application of MIP as SPE sorbents was desired to eliminate the interferences of sample matrix and get cleaner extracts than the traditional SPE, which resulted in a more selective and reliable analytical protocol.

Various strategies could be used to prepare MIP for this purpose including precipitation polymerization, bulk polymerization, suspension polymerization, swelling polymerization, miniemulsion polymerization, and core-shell emulsion polymerization [16-18]. The Knopp group has ever prepared molecular imprinted microspheres $(>3 \mu \mathrm{m})$ and nanospheres $(\approx 450 \mathrm{~nm})$ for $\operatorname{di}(2$-ethylhexyl)phthalate (DEHP) by precipitation polymerization. When used as HPLC stationary phase, the microspheres $(>3 \mu \mathrm{m})$ exhibited strong affinity for the template DEHP with an imprinted factor (IF) higher than 8.0 [19]. Recently, Yan et al. synthesized a new imprinted microsphere of diisononyl phthalate via precipitation polymerization and used it for the simultaneous determination of the PAEs from plastic bottled beverages. The average recoveries of the five PAEs at three spiked levels ranged from 84.3 to $96.2 \%$ [20]. Qi et al. optimized different porogens and functional monomers to prepare an MIP of monobutyl phthalate, which was used for the preconcentration and determination of monobutyl phthalate from the bottled water by MIP-SPE-HPLC method [21]. An MI-SPME fiber of dibutyl phthalate (DBP) was prepared into a special device by bulk polymerization and was applied to the pretreatment of five kinds of phthalates dissolved in spiked aqueous samples, followed by the determination by (GC/MS) [22]. This method was also used to synthesize the MI-SPE sorbents using dibutyl phthalate (DBP) as a template molecule; the resulted powders with particle sizes $35-75 \mu \mathrm{m}$ were evaluated and used for selective SPE of DBP from soybean milk [23]. Recently, Shaikh et al. reported a similar MIP selective for DEHP, which was prepared by suspension polymerization using methacrylamide as a functional monomer and N,N-methylene-bis-acrylamide as a crosslinker. The imprinted polymer was employed for solid-phase extraction of DEHP from water samples prior to GC determination [24].

In this paper, synthesis of a noncovalently imprinted MIP using di-n-octylphthalate (DOP) as a template was firstly carried out by bulk polymerization. The obtained MIP was applied as an SPE sorbent to preconcentrate and determine the PAEs in the bottled sprites by HPLC, and better recoveries in three spiked levels were obtained under the optimal conditions, compared with the commercial SPE cartridge used for the enrichment of PAEs.

\section{Experimental}

2.1. Instruments and Chemicals. Chromatographic separation was performed by an Easy Sep 1020LC HPLC system from Shanghai Unimicro Technologies, China, equipped with a single pump, an injector with a $20 \mu \mathrm{L}$ quantitative tube (Rheodyne 7725i) and a UV detector with a changeable wavelength in the range of $190 \sim 600 \mathrm{~nm}$. A Kromasil $\mathrm{C}_{18}$ column $(150 \mathrm{~mm} \times 4.6 \mathrm{~mm}$ i.d., particle size $5 \mu \mathrm{m})$ was used as the stationary phase. Binding experiments were performed using a UV-2100 spectrophotometer (Beijing Beifen-Ruili Analytical Instrument Co. Ltd.), Pressure Blowing Concentrator (Supelco, USA), SPE Manifold (Supelco, USA), TENSOR27 infrared spectrometer (BRUKER, Germany), and Quanta 200 scanning electronic microscope (FEI, Hillsboro, OR, USA).

Four phthalates (DMP, DEP, DBP, and DOP) were purchased from Tianjin Fuchen Chemical Reagent Co., and MAA, EDMA, AIBN, acetonitrile (ACN), and acetic acid (AA) were purchased from Tianjin Guangfu Institute of Chemicals. The monomers were purified by distillation to remove the inhibitors. All other chemicals used are of chromatographically pure and analytical grade. ProElut PLS Glass Cartridge $(60 \mathrm{mg} / 3 \mathrm{~mL})$ was purchased from Dikma Technologies Inc., CA, USA.

2.2. Preparation of MIP. Some previous reports of the synthesis of MIP about the similar template were referred to for our preparation studies with some modification of the choice of different porogens and templates [19-24]. Schematic illustration of DOP molecular imprinting procedures and the structurally related compounds of DOP for the experiments were shown, respectively, in Figures 1(a) and 1(b). Briefly, the molar ratio of template to monomer was selected at $1: 4$ to ensure the formation of defined recognition sites with polymers. Briefly, MIP is synthesized using bulk polymerization method by dissolving $1 \mathrm{mmol}$ DOP in a glass tube with $10 \mathrm{~mL} \mathrm{ACN}$, followed by ultrasonication about $30 \mathrm{~min}$ to form a stable complex of imprint molecule and monomers. The obtained solution was then cooled at room temperature, followed by adding $20.0 \mathrm{mmol}$ of EDMA, $30 \mathrm{mg}$ AIBN. Then, the prepared sample in the tube was degassed and purged with nitrogen before being sealed with a septum. The solutions were further saturated with dry nitrogen for $5 \mathrm{~min}$, and the glass tubes were placed in a water bath at $60^{\circ} \mathrm{C}$ for $24 \mathrm{~h}$. After polymerization, the tubes were broken, the obtained polymers were ground and sieved, and the particles with sizes $47-74 \mu \mathrm{m}$ were collected and then repeatedly suspended in acetone to remove the small particles for next use. The template was removed by the Soxhlet extraction using a two-step procedure, being washed by acetic acid and methanol (1:9, v/v) about $12 \mathrm{~h}$ as a first step, then by methanol for $6 \mathrm{~h}$ as a second step. The prepared polymers were dried at $60^{\circ} \mathrm{C}$ for $24 \mathrm{~h}$ under vacuum and stored at desiccators prior to use. Nonimprinted polymers (NIPs) were synthesized and treated under the same conditions but without the addition of the template.

2.3. Equilibrium Rebinding and Binding Selectivity of MIP. Steady-state binding studies were carried out by UV spectrophotometry [20-24]. The standard DOP solutions were 


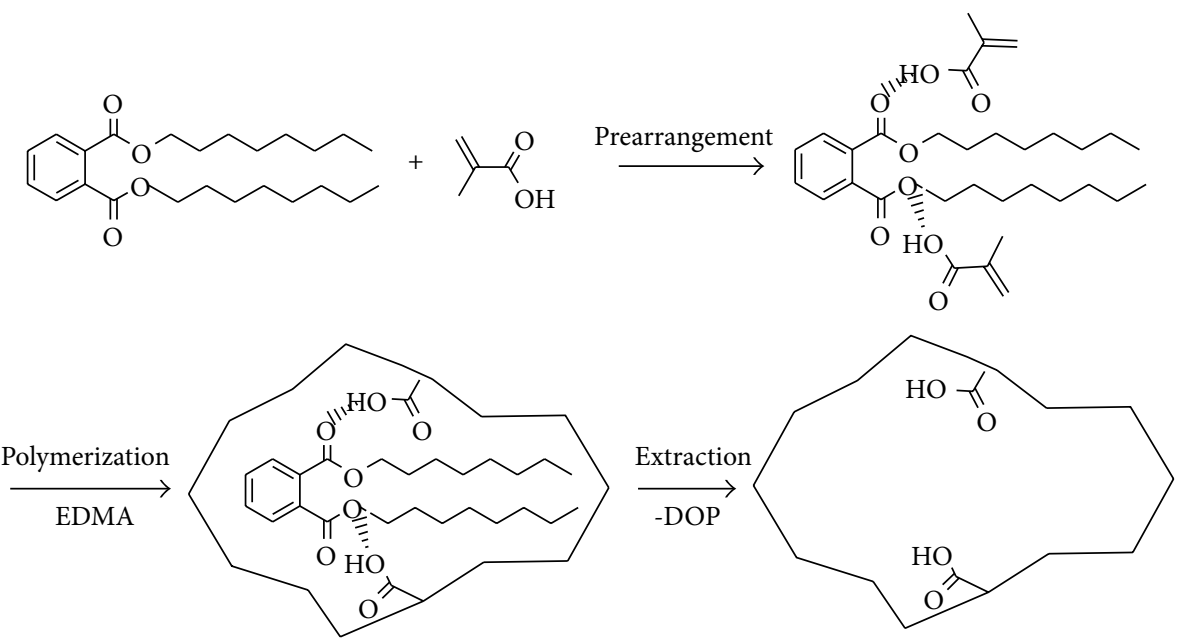

(a)<smiles>COC(=O)c1ccccc1C(=O)OC</smiles>

DMP<smiles>CCCCOC(=O)c1ccccc1C(=O)OCCCC</smiles><smiles>CCOC(=O)c1ccccc1C(=O)OCC</smiles>

DEP<smiles>CCCCCCCCCCCOC(=O)c1ccccc1C(=O)OCCCCC</smiles>

(b)

FIGURE 1: (a) Schematic illustrations of the imprint formation and molecular recognition. (b) Molecular structures of the related compounds.

prepared in $\mathrm{ACN}$, and the concentrations of DOP in the solutions varied in the range of $0.15-1.0 \mathrm{mmol} / \mathrm{L}$. In a typical experiment, an amount of $20.0 \mathrm{mg}$ of both polymers (MIP and NIP) was weighed and put into a $10 \mathrm{~mL}$ vial, respectively; $2 \mathrm{~mL}$ of DOP standard solution with different concentrations was then mixed with each polymer, respectively. Afterwards, the mixture was shaken with a horizontal shaker for $24 \mathrm{~h}$ at room temperature, followed by centrifugation at $3000 \mathrm{rpm}$ for $5 \mathrm{~min}$. The suspension was filtered by a filter with $0.22 \mu \mathrm{m}$ pore size, and the filtrated solution was diluted and analyzed by UV spectrometry to obtain the concentration of DOP. The amount of DOP bound to the polymer $(Q)$ was calculated by subtracting the amount of free DOP from the initial amount added to the mixture $\left(C_{0}\right)$. The absorption quantity $(Q)$ was calculated by [19-24] $Q=\left(C_{0}-C\right) \times V / W$, where $C_{0}$ is the template DOP concentrations in the initial solution and $C$ is that of free template in the solution containing imprinted polymer after shaken for $24 \mathrm{~h}$. Volume of bulk solution is $V$, and $W$ is the weight of the dry polymer used.

The same experiments were performed using other structurally related compounds (DMP, DEP, and DBP). Briefly, an amount of $20.0 \mathrm{mg}$ of both polymers (MIP and NIP) was weighed and put into a $10 \mathrm{~mL}$ vial, respectively; then
$2 \mathrm{~mL}$ of DOP standard solution with constant concentration of $0.7 \mathrm{mmol} / \mathrm{L}$ was mixed with each polymer, respectively. The data of the static absorption experiment was further processed with the Scatchard equation to estimate the binding parameters of the MIP and NIP [18-20]. The Scatchard equation was described as follows: $Q / C=\left(Q_{\max }-Q\right) / K_{d}$. Here, $K_{d}$ and $Q_{\max }$ were balance dissociation constant and maximum binding amount, respectively, and $C$ was the balance concentration of the target molecule. The values of $K_{d}$ and $Q_{\max }$ were calculated from the slope and intercept of the linear line plotted in $Q / C$ versus $Q$.

2.4. Preparation of MI-SPE Cartridge. MI-SPE cartridge was prepared with empty $3 \mathrm{~mL}$ cartridges (DIKMA Sci. \& Tech.) packed with $60 \mathrm{mg}$ polymers $(47-74 \mu \mathrm{m})$ as a sorbent and $20 \mathrm{mg}$ diatomite as a filter aid, respectively. Each cartridge was attached to two sieve plates at the bottom and the top end, respectively. The cartridges were subjected to vacuum for $30 \mathrm{~s}$ before insertion of a second frit on top of the sorbent bed.

2.5. Analysis of Real Samples. The sprite beverage packed in plastic cups was initially sonicated and filtered by a filter with 
$0.22 \mathrm{~m}$ pore size. $5 \mathrm{~mL}$ of the sample solution or spiked samples were then flown through MI-SPE cartridge at flow rate of $1.0 \mathrm{~mL} / \mathrm{min}$, respectively. After the cartridge was washed with $5.0 \mathrm{~mL}$ of water (contains $10 \%$ methanol), $3.0 \mathrm{~mL}$ of methanol was flown through the cartridge to carry out the absorbed organics in MIP. The collected solution was dried under $\mathrm{N}_{2}$ flow, and the residues were recovered into $5 \mathrm{~mL}$ of $\mathrm{ACN}$ for HPLC analysis. Prior to each extraction, the cartridges should be conditioned with $3 \mathrm{~mL} \mathrm{MeOH}$ and water, respectively. The ProElut PLS Glass Cartridge was processed according to the application manual about the pretreatment of PAEs from Dikma Technologies Inc. It was firstly conditioned with $3 \mathrm{~mL}$ $\mathrm{MeOH}$ and $3 \mathrm{~mL} \mathrm{H}_{2} \mathrm{O}$; then the samples passed through the cartridge with a constant flow rate of $1 \mathrm{~mL} / \mathrm{min}$, and $3 \mathrm{~mL} 5 \%$ $\mathrm{MeOH}$ aqueous solution was used to wash, followed by the elution procedure with $3 \mathrm{~mL} \mathrm{MeOH}$.

\section{Results and Discussions}

3.1. Characterization of SEM and IR for MIP and NIP. Figure 2 presented the SEM images of prepared polymers. Generally, polymers without templates displayed a relatively smooth surface, whilst there were some speckles and cavities appearing in the resulted MIP. The obvious difference between NIP and their relative MIP was definitely attributed to imprinting effect. After the DOP template embedded in the framework of the polymers was removed, thereby more speckles and bigger cavities were formed, compared with NIP.

The FT-IR spectra (KBr pellet) of the prepared polymers, the monomer (MAA), the crosslinker (EDMA), and template (DOP) coating were shown in Figure 3, respectively. Obvious diference about absorption bands could be found in the range of $1100-1800 \mathrm{~cm}^{-1}$ between the two polymers (MIP and NIP) and other three compounds (MAA, EDMA and DOP). The main functional groups of the predicted MIP and NIP coatings could be found with corresponding infrared absorption peaks. A broad absorption band at $3446 \mathrm{~cm}^{-1}$ on the MIP coating corresponded to several overlapped peaks of infrared absorption such as the stretching vibration of $\mathrm{O}-\mathrm{H}$ bonds of MAA molecules (functional monomer), the hydrogen bonding, and electrostatic binding interactions between DOP and MAA. However, for NIP, just a weaker absorption peak around $3446 \mathrm{~cm}^{-1}$ was discovered because of the lack of hydrogen bonding or electrostatic binding interaction between DOP and MAA. Moreover, no obvious difference of other peeks could be observed in these spectra in the range of $1158-2992 \mathrm{~cm}^{-1}$. It was attributed that almost all templates are removed for MIP. Other absorption peaks matched both MIP and NIP coatings as follows: 2960 or $2970 \mathrm{~cm}^{-1}$ (stretching vibration of $\mathrm{C}-\mathrm{H}$ bonds on methyl groups); 1731 or $17331 \mathrm{~cm}^{-1}$ (stretching vibration of $\mathrm{C}=\mathrm{O}$ bonds on carbonyl groups); 1158 or $1159 \mathrm{~cm}^{-1}$ (stretching vibration of $\mathrm{C}-\mathrm{O}$ and $\mathrm{C}-\mathrm{C}$ ).

3.2. Equilibrium Rebinding Study and the Scatchard Analysis. Figure 4(a) shows the binding capacity of MIP (and NIP) for DOP. It is easily understood that the MIP exhibited a higher capacity for DOP than that of the NIPs. Herein, the amount of DOP absorbed by both MIP and NIP was increased in the selective concentration $(0.15-1.0 \mathrm{mmol} / \mathrm{L})$ of DOP, whilst the amount of DOP absorbed on NIP was lower compared with that in MIP. For example, the $Q$ values of MIP and NIP were $20.4 \mu \mathrm{mol} / \mathrm{g}$ and $13.7 \mu \mathrm{mol} / \mathrm{g}$ at the concentration of $0.5 \mathrm{mmol} / \mathrm{L}$, but obvious changes were obtained with a $Q$ value of $31.8 \mu \mathrm{mol} / \mathrm{g}$ and $17.3 \mu \mathrm{mol} / \mathrm{g}$, respectively. It was attributed that the MIP possessed specific binding for DOP, and NIP did not. Furthermore, the rebinding isotherm of DOP on MIP and NIP in ACN showed that both specific and nonspecific absorptions of DOP increased with higher concentration of DOP solution.

The Scatchard analysis suggested that DOP was recognized by the prepared MIP with two classes of binding sites. It was illustrated that MIP for DOP binding sites were nonuniform as shown in Figure 4(b). Two straight lines could be drawn, indicating that the affinities of the binding sites in MIP are heterogeneous and could be approximated by two dissociation constants corresponding to the high- and low-affinity binding sites for MIP and NIP, respectively. For the higher affinity site of MIP, its linear equation is $Q / C=$ $3436-213.6 Q$, and the values of $K_{d}$ and $Q_{\max }$, calculated from the slope and intercept of the linear portion of the Scatchard analysis, are $0.0047 \mu \mathrm{mol} / \mathrm{mL}$ and $16.09 \mu \mathrm{mol} / \mathrm{g}$, respectively. For the lower affinity site, the linear equation is $Q / C=121.75-2.41 Q ; K_{d}$ and $Q_{\max }$ are $0.42 \mu \mathrm{mol} / \mathrm{g}$ and $50.60 \mu \mathrm{mol} / \mathrm{g}$, respectively. In contrast, for the higher affinity site of NIP, its linear equation is $\mathrm{Q} / \mathrm{C}=6076-467.5 \mathrm{Q}$, and the values of $K_{d}$ and $Q_{\max }$ are 0.0021 and $13.00 \mu \mathrm{mol} / \mathrm{g}$, respectively. For the lower affinity site, the linear equation is $Q / C=94.41-4.18 Q$, and $K_{d}$ and $Q_{\max }$ are $0.239 \mu \mathrm{mol} / \mathrm{mL}$ and $22.57 \mu \mathrm{mol} / \mathrm{g}$, respectively.

3.3. Specificity Study for the Prepared Polymer. PAEs with shorter alkyl chains (e.g., DMP, DEP, and DBP) were more easily utilized than the PAEs with longer alkyl-chains (DOP) in production processes. Herein, the ability of MIP to discriminate DOP and other three structurally related compounds was assessed in single-analyte binding assays. After equilibrium binding experiments were performed according the foregoing procedures, the relative $Q_{\text {MIP }}$ and $Q_{\text {NIP }}$ for each analyte could be obtained, respectively. The imprinting factor expresses the ratio of specific-to-nonspecific binding for each compound $\left(\mathrm{IF}=Q_{\mathrm{MIP}} / Q_{\mathrm{NIP}}\right)$. In each case, analyte uptake was normalized against the levels of template DOP absorbed and expressed as selectivity index (SI) calculated according to [25-28] SI = IF analyte $/ \mathrm{IF}_{\text {template. }}$. When SI is equal to 1 , there is no difference between MIP and the control polymer. Obviously, the bindings of the DMP, DEP, DBP, and DOP in MIP are all higher than that in NIP, indicating the higher specific bindings for the tested compounds in MIP. These are due to the high similarity of the molecular structures between DMP, DEP, DBP, and DOP.

Gradual decreased $Q_{M I P}$ to the target analyte could be obtained for PAEs with shorter alkyl-chains, whilst $Q_{\mathrm{NIP}}$ to the target molecular gradually increased except for DOP template molecule. These experimental results demonstrated that the imprinting was primarily based on the interaction 


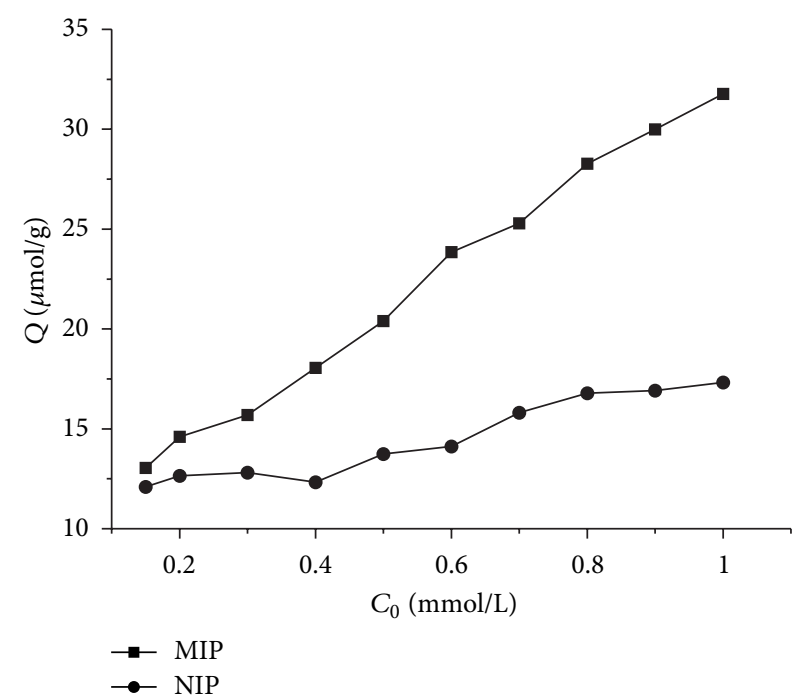

(a)

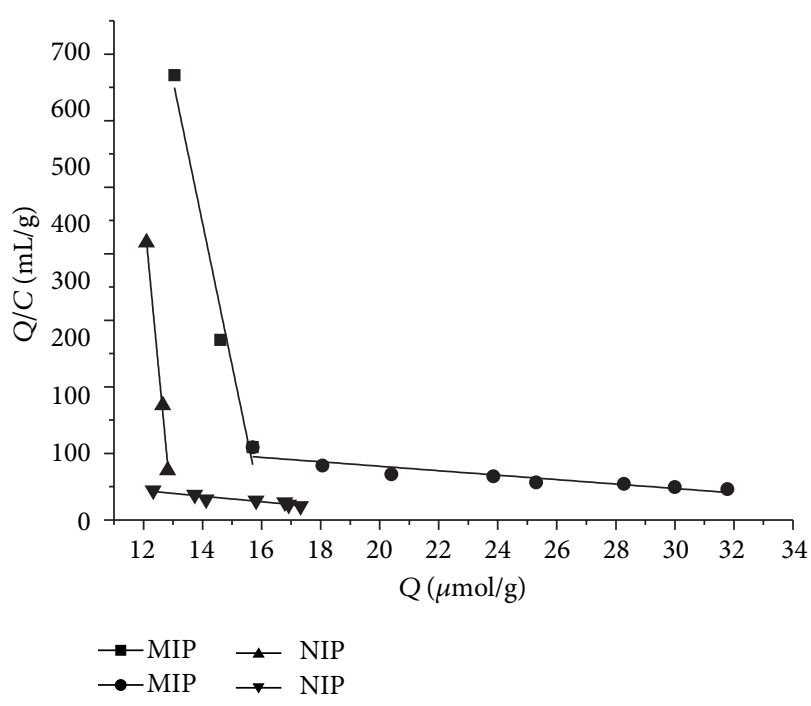

(b)

FIgURE 2: (a) Binding isotherm of DOP on MIP and NIP in water. (b) The Scatchard plots to estimate the binding nature of MIP and NIP.

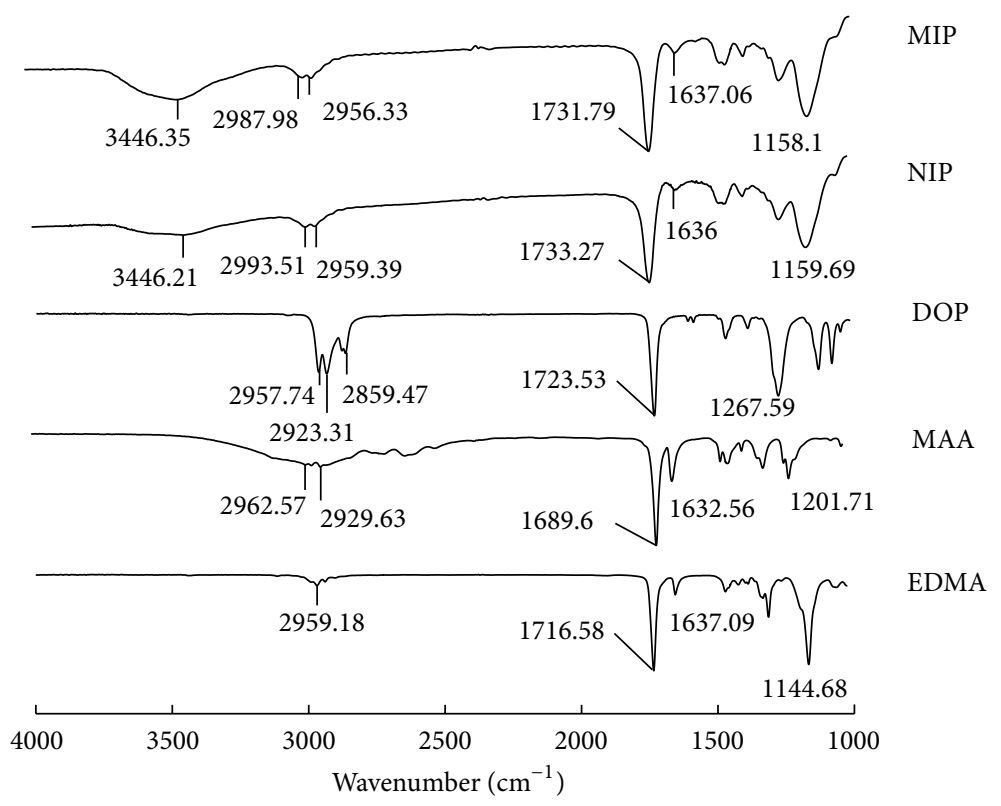

FIgURE 3: FT-IR spectra of MAA, EDMA, DOP, MIP, and NIP.

of analyte with functional groups within polymer cavities, but the combined effect of shape and size complementarity probably affected the imprinting efficiency effectively. It is easily understood that the largest values of $\alpha$ and SI were achieved for the template molecule (DOP), compared to other structurally related compounds with similar values of $\alpha$ and SI. These IF and SI values obtained are comparatively given in Table 1.

3.4. Optimization of MI-SPE Cartridge. The MI-SPE procedures require the same steps used in a common SPE procedure: conditioning, sample loading, washing, and elution.
Factors that probably influence the extraction process, such as loading solvent, $\mathrm{pH}$, flow rate and volume of loading solution, washing solvent, and amount of the eluting solvent, should be evaluated to achieve the highest extraction efficiency, respectively.

The loading solvent needs to be carefully selected to promote the rebinding of the substrate to the specific sites. In our experiments, when methanol, $\mathrm{ACN}$, and ethyl acetate were selected as the loading solvents, respectively, more than $90 \%$ DOP could not be retained on the MI-SPE cartridge. Whilst when dichloromethane and hexane were selected for it, slight swelling would occur for the packed adsorbent. Since 


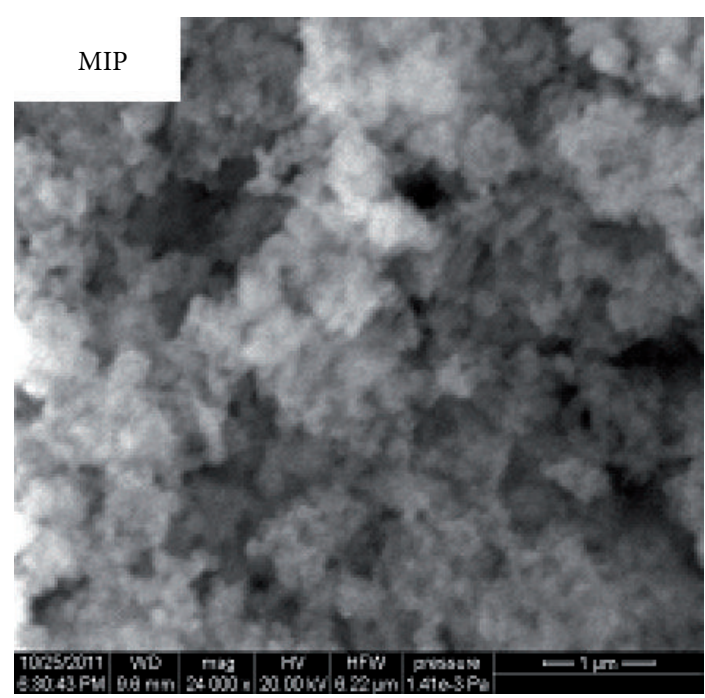

(a)

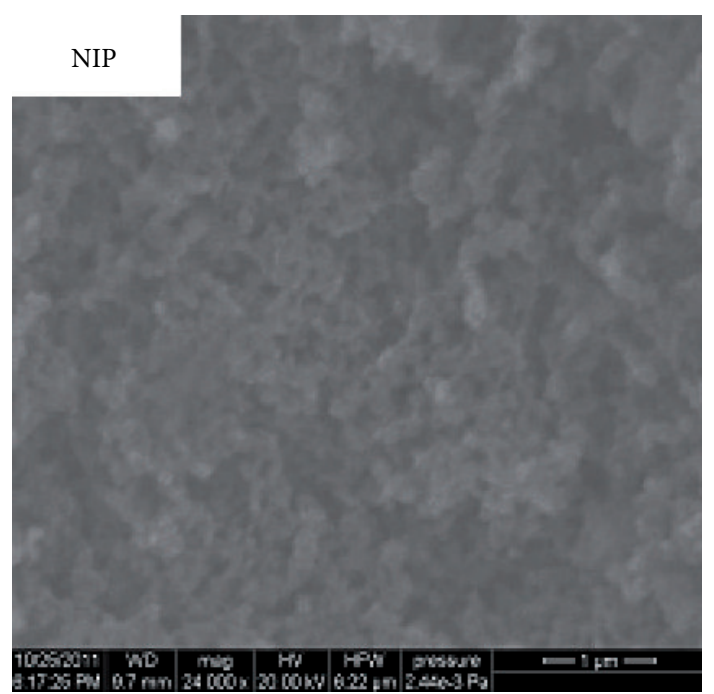

(b)

FIGURE 4: SEM figures of the prepared MIP and NIP.

TABLE 1: Maximum binding number $\left(Q_{\max }\right)$, imprinting factors (IF), and standardized selectivity (SI) for the structurally related compounds of DOP.

\begin{tabular}{lcccc}
\hline Analytes & $Q_{\mathrm{MIP}}(\mu \mathrm{mol} / \mathrm{g})$ & $Q_{\mathrm{NIP}}(\mu \mathrm{mol} / \mathrm{g})$ & $\mathrm{IF}$ & $\mathrm{SI}$ \\
\hline DOP & 27.05 & 12.93 & 2.09 & 1.00 \\
DMP & 19.02 & 15.86 & 1.19 & 0.83 \\
DEP & 20.33 & 16.52 & 1.23 & 0.81 \\
\hline DBP & 24.76 & 19.29 & 1.28 & 0.77 \\
\hline
\end{tabular}

most of environmental samples are in aqueous solutions and the real beverage analysis was carried out in water, $5 \mathrm{~mL}$ water with $10 \mathrm{mg} / \mathrm{L}$ DOP was chosen as the loading solvent. In this case, none of DOP was observed in the effluent liquids of the MI-SPE cartridge, which revealed the good affinity of the prepared MIP to the target analyte of DOP. Different flow rates of loading solvent were investigated in the range of 1.0 to $5.0 \mathrm{~mL} / \mathrm{min}$, and the results displayed that the recoveries of DOP decreased to about $40 \%$ when the flow rate was changed to $5.0 \mathrm{~mL} / \mathrm{min}$ (see Figure 5). Considering the balance between the larger recovery of DOP and the total analysis time, the flow rate of $1.0 \mathrm{~mL} / \mathrm{min}$ was used in the throughout optimization.

Choice of washing solvent aims at selective extraction of the specific bound DOP. Loading spiked aqueous solution can make the target analyte retained on the SPE cartridge through both specific and nonspecific interactions, such as hydrophobic interaction. Thus, a subsequent washing process is required to remove the nonspecifically bound compound and consequently to achieve selective preconcentration of DOP. $5 \mathrm{~mL}$ water containing different percentages of $\mathrm{MeOH}$ $(0 \%, 10 \%, 20 \%, 30 \%$, and $50 \%$, resp.) was tested for washing conditions. The results showed that the recovery of DOP decreased obviously with the increase of $\mathrm{MeOH}$ content. Pure water possessed the highest elution strength, which resulted

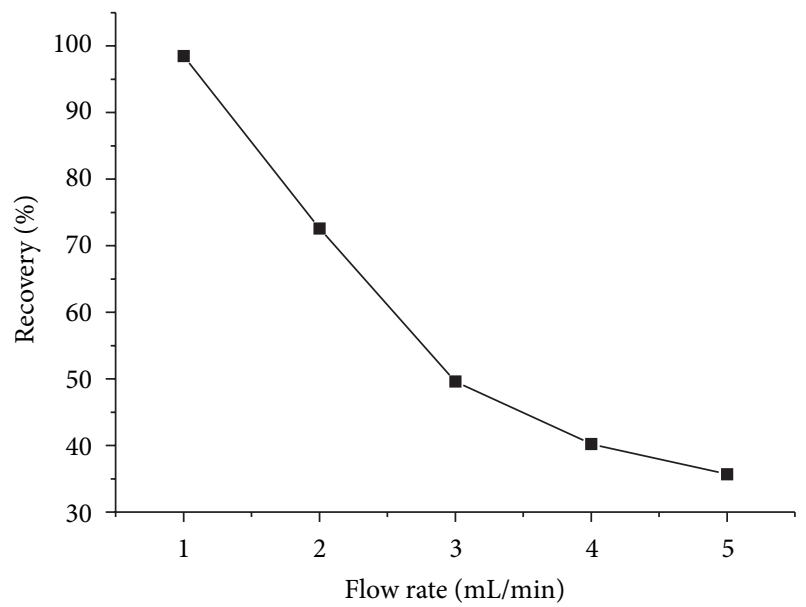

FIgURE 5: Optimization of the flow rates of loading solvent.

in the best recoveries of DOP (nearly 100\%). Thus, water was chosen as the washing solution in the experiments.

It is necessary to find that a suitable solvent elutes the PAEs from the MI-SPE cartridge before HPLC analysis; so different types of solutions including MeOH-AA $(8: 2$, $\mathrm{v} / \mathrm{v})$, and $\mathrm{MeOH}-\mathrm{AA}(9: 1, \mathrm{v} / \mathrm{v}), \mathrm{MeOH}$ were selected to investigate their elution efficiencies. The results indicated that both $\mathrm{MeOH}-\mathrm{AA}(8: 2, \mathrm{v} / \mathrm{v})$ and $\mathrm{MeOH}-\mathrm{AA}(9: 1, \mathrm{v} / \mathrm{v})$ had relatively lower eluting ability for all PAEs, only fewer target analytes were eluted out with more than $15 \mathrm{~mL}$ elution solvent, and pure $\mathrm{MeOH}$ provided the highest efficiency, which is possibly due to the interactions between the PAEs and the MIPs disrupted under this condition. Thus, on the basis of the elution efficiency and minimum solvent consumption, $3 \mathrm{~mL}$ of $\mathrm{MeOH}$ was adopted to elution of the PAEs from the cartridges. Therefore, the volume of methanol was determined as $3 \mathrm{~mL}$ in all the experiments. $\mathrm{PH}$ value 
TABLE 2: Recoveries of PAEs in spiked beverages by MIP, NIP, and PLS cartridges in three spiked samples $(n=5)$.

\begin{tabular}{|c|c|c|c|c|c|c|c|}
\hline \multirow{2}{*}{ Cartridge } & \multirow{2}{*}{ PAEs } & \multicolumn{2}{|c|}{ Spiked level I } & \multicolumn{2}{|c|}{ Spiked level II } & \multicolumn{2}{|c|}{ Spiked level III } \\
\hline & & Recovery (\%) & RSD (\%) & Recovery (\%) & RSD (\%) & Recovery (\%) & RSD (\%) \\
\hline \multirow{3}{*}{ MIP } & DMP & 91.5 & 2.9 & 92.0 & 3.6 & 90.4 & 3.4 \\
\hline & DBP & 92.2 & 1.6 & 92.8 & 3.8 & 93.9 & 2.6 \\
\hline & DOP & 96.2 & 3.2 & 96.8 & 2.9 & 97.8 & 1.9 \\
\hline \multirow{3}{*}{ NIP } & DMP & 4.9 & 1.9 & 5.0 & 2.2 & 5.2 & 1.6 \\
\hline & $\mathrm{DBP}$ & 1.8 & 1.6 & 2.1 & 2.4 & 2.0 & 1.7 \\
\hline & DOP & 2.6 & 4.0 & 2.8 & 3.7 & 2.9 & 2.2 \\
\hline \multirow{3}{*}{ PLS } & DMP & 84.9 & 5.2 & 85.6 & 4.6 & 88.9 & 1.4 \\
\hline & DBP & 82.0 & 3.9 & 84.3 & 4.8 & 87.5 & 2.5 \\
\hline & DOP & 80.2 & 4.4 & 83.2 & 3.9 & 85.1 & 3.0 \\
\hline
\end{tabular}

(I) Added: $0.3 \mathrm{mg} / \mathrm{L}$ DMP, and $0.3 \mathrm{mg} / \mathrm{L}$ DBP, $0.3 \mathrm{mg} / \mathrm{L}$ DOP; (II) added: $0.5 \mathrm{mg} / \mathrm{L}$ DMP, and 0.5 mg/L DBP, $0.5 \mathrm{mg} / \mathrm{L}$ DOP; (III) added: $1.0 \mathrm{mg} / \mathrm{L}$ DMP, $1.0 \mathrm{mg} / \mathrm{L}$ $\mathrm{DBP}$, and $1.0 \mathrm{mg} / \mathrm{L}$ DOP.

of loading solution may influence the recovery of MIP-SPE. According to the previous reports about PAEs extraction, the $\mathrm{pH}$ of loading sample was usually set at 6.0 in the optimum experiment. Herein, the samples with $\mathrm{pH}$ values about 6.0 were not further optimized in our experiments.

The evaluation of the cartridge capacity was carried out by passing increasing step volume of $1 \mathrm{~mL}$ standard solution with a constant concentration of DOP $(10 \mathrm{mg} / \mathrm{L})$ through the MI-SPE. The results showed that DOP could be detected after $65 \mathrm{~mL}$ standard solution passed through the MI-SPE cartridge; so the largest cartridge capacity could be calculated to be $2.78 \times 10^{-3} \mathrm{mmol} / \mathrm{g}$.

3.5. Validation of the MI-SPE-HPLC Method. The calibration curve was constructed using the areas of the chromatographic peaks measured at six increasing spiked levels ranging from 0.1 to $10 \mathrm{mg} / \mathrm{L}$. Good linearity was observed for three analytes throughout the concentration range, and the regression equations are calculated as follows: $y_{\mathrm{DEP}}=4.27 \times 10^{5} x+$ $2.12 \times 10^{5}(r=0.9998, \mathrm{LOD}=0.008 \mathrm{mg} / \mathrm{L}) ; y_{\mathrm{DBP}}=2.91 \times$ $10^{5} x+1.65 \times 10^{5}(r=0.9992, \mathrm{LOD}=0.012 \mathrm{mg} / \mathrm{L}) ; y_{\mathrm{DOP}}=$ $2.47 \times 10^{5} x+1.51 \times 10^{5}(r=0.9980, \mathrm{LOD}=0.018 \mathrm{mg} / \mathrm{L})$.

To further investigate the effect of sample matrix on the selectivity and accuracy of the MI-SPE-HPLC method for real analysis, the recovery experiments were carried out by spiking three different levels of three PAEs into the beverage samples. The accuracy and precision of the method were assessed by performing analyses of spiked samples in five replicates. As shown in Table 2, the relative standard deviations (RSDs) for all recoveries were in a range of $1.4-5.2 \%$. The highest recoveries (90.4-97.8\%) and clear chromatogram of MI-SPE-HPLC demonstrated that MIP can be used as the group-recognition sorbents in SPE for trace isolation of the PAEs in beverage samples, which overcome the drawbacks of template leakage of MIP in real analysis. Under the same experimental conditions, less recoveries of $1.8-4.9 \%$ were only achieved using NIP as the sorbent, demonstrating lower affinity of itself towards the PAEs. It resulted from the specific recognition sites of MIP formed by the imprinting effect. Moreover, commercial ProElut PLS cartridge provided lower recoveries for PAEs (80.2-88.9\%), which was due to its lower affinities and less specific identification to the analytes. ProElut PLS is a highly cross-linked polystyrenedivinylbenzene (PS-DVB) copolymer used for extraction of polar analytes in aqueous solvents where traditional $\mathrm{C}_{18}$ and $\mathrm{C}_{8}$ sorbents are not advisable because they are not "wettable." It is ideal in screening applications where a broad range of analytes can be extracted including PAEs, PAHs, and phenols. Here, the results showed that a better purifying effect was obtained for the MIP cartridge than commercial PLS cartridge, and all of the interferences in sample matrix could be effectively eliminated. All of the above indicated that the MI-SPE-HPLC method has the advantages of simplicity, rapidity, and sensitivity and could be potentially applied for the monitoring of PAEs residues in beverage products.

3.6. Sample Analysis of Plastic Bottled Beverage Products. Blank sprite samples were investigated as a reference, and no peaks of PAEs in chromatogram were observed at the same retention time, compared with the standard solution, but there were several impurities peaks for the original sprite sample (see Figures 6(a) and 6(b)). The spiked sample was then selected to pass through the PLS, NIP, and cartridges MIP, respectively, and the chromatograms in Figures 6(c), $6(\mathrm{~d})$, and $6(\mathrm{e})$ indicated that there were no noticeable interferences from the matrix, the interferences were significantly reduced after SPE enrichments. Moreover, the largest peak areas for three PAEs were obtained for MIP cartridge and were high recoveries than those of NIP and PLS, which revealed that the MIP had high affinity and selectivity for PAEs in aqueous solution.

\section{Conclusions}

In this study, A MIP was prepared by bulk polymerization techniques and characterized by SEM and FT-IR. Equilibrium rebinding studies demonstrated that MIP possessed specific binding for the template, compared with NIP. The Scatchard plot suggested a two-site binding behavior, and MIP had high rebinding capability for other structural 


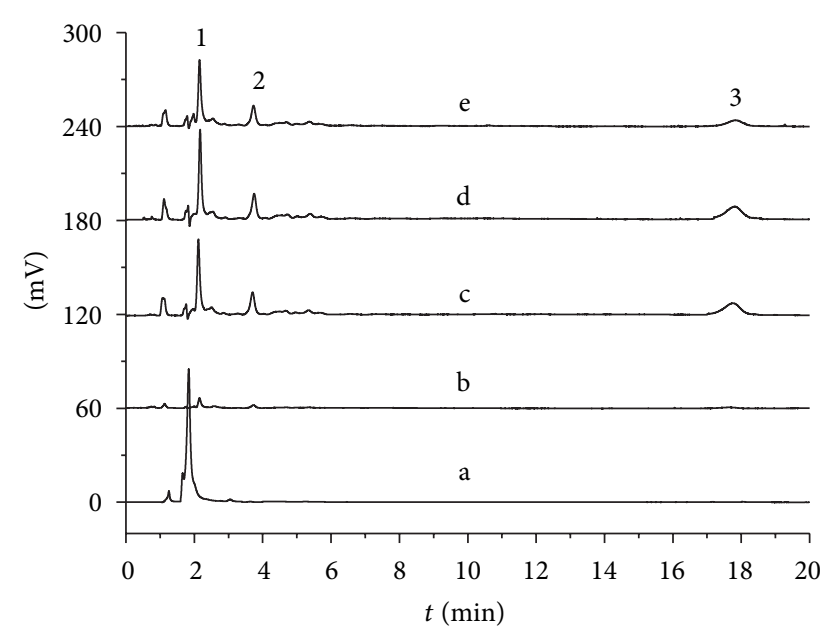

FIGURE 6: Comparative chromatograms obtained by analysis of the bottled sprite samples. Experimental conditions: mobile phase $\left(\mathrm{ACN} / \mathrm{H}_{2} \mathrm{O}=95 / 5, \mathrm{v} / \mathrm{v}\right)$, flow rate $=0.8 \mathrm{~mL} / \mathrm{min}, \lambda=224 \mathrm{~nm}$, and injection volume $=20 \mu \mathrm{L}$. Peak orders: $1:$ DMP, 2: DBP, and 3: DOP; Symbols: a: original sprite sample without pretreatment, b: spited sample through NIP cartridge after spiting with DMP, DBP, and DOP at $0.3,0.3$, and $0.3 \mathrm{mg} / \mathrm{L}$, respectively, c: sprite sample through MIP cartridge after spiting with DMP, DBP, and DOP at $0.3,0.3$, and $0.3 \mathrm{mg} / \mathrm{L}$, respectively. d: standard solution, e: spited sample through PLS cartridge after spiting with DMP, DBP, and DOP at $0.3,0.3$, and $0.3 \mathrm{mg} / \mathrm{L}$, respectively.

analogs. Sample loading, washing, and desorption conditions were also optimized, followed by the determination of PAEs by MI-SPE-HPLC. Good precision and accuracy of the MISPE cartridge for DOP and related compounds in spiked bottled beverage demonstrated the feasibility of the prepared MIP for extraction of phthalates.

\section{Acknowledgments}

The financial support by the Scientific Research Foundation for the Returned Overseas Chinese Scholars, State Education Ministry, and the National Natural Science Foundation of China (nos. 30070506 and 30771436) are gratefully acknowledged.

\section{References}

[1] J. Nishioka, C. Iwahara, M. Kawasaki et al., "Di-(2-ethylhexyl) phthalate induces production of inflammatory molecules in human macrophages," Inflammation Research, vol. 61, pp. 6978, 2012.

[2] F. Zeng, K. Cui, Z. Xie et al., "Phthalate esters (PAEs): emerging organic contaminants in agricultural soils in peri-urban areas around Guangzhou, China," Environmental Pollution, vol. 156, no. 2, pp. 425-434, 2008.

[3] I. Ostrovský, R. Čabala, R. Kubinec et al., "Determination of phthalate sum in fatty food by gas chromatography," Food Chemistry, vol. 124, no. 1, pp. 392-395, 2011.
[4] A. Mehdinia, F. Roohi, and A. Jabbari, "Rapid magnetic solid phase extraction with in situ derivatization of methylmercury in seawater by $\mathrm{Fe}_{3} \mathrm{O}_{4}$ /polyaniline nanoparticle," Journal of Chromatography A, vol. 1218, no. 28, pp. 4269-4274, 2011.

[5] H. Zhang, W. P. Low, and H. K. Lee, "Evaluation of sulfonated graphene sheets as sorbent for micro-solid-phase extraction combined with gas chromatographymass spectrometry," Journal of Chromatography A, vol. 1233, pp. 16-21, 2012.

[6] Q. Liu, J. Shi, L. Zeng, T. Wang, Y. Cai, and G. Jiang, "Evaluation of graphene as an advantageous adsorbent for solid-phase extraction with chlorophenols as model analytes," Journal of Chromatography A, vol. 1218, no. 2, pp. 197-204, 2011.

[7] R. A. Anderson, M. M. Ariffin, P. A. G. Cormack, and E. I. Miller, "Comparison of molecularly imprinted solid-phase extraction (MISPE) with classical solid-phase extraction (SPE) for the detection of benzodiazepines in post-mortem hair samples," Forensic Science International, vol. 174, no. 1, pp. 4046, 2008.

[8] E. Rodriguez, M. C. Moreno-Bondi, and M. D. Marazuela, "Multiresidue determination of fluoroquinolone antimicrobials in baby foods by liquid chromatography," Food Chemistry, vol. 127, no. 3, pp. 1354-1360, 2011.

[9] H. Yan, M. Tian, and K. H. Row, "Selective solid-phase extraction of glabridin from licorice root using molecularly imprinted polymer," Separation Science and Technology, vol. 44, no. 2, pp. 359-369, 2009.

[10] C. Blasco, P. Vazquez-Roig, M. Onghena, A. Masia, and Y. Picó, "Analysis of insecticides in honey by liquid chromatographyion trap-mass spectrometry: comparison of different extraction procedures," Journal of Chromatography A, vol. 1218, no. 30, pp. 4892-4901, 2011.

[11] L. Vidal, M. Riekkola, and A. Canals, "Ionic liquid-modified materials for solid-phase extraction and separation: a review," Analytica Chimica Acta, vol. 715, pp. 19-41, 2012.

[12] R. Ramautar, G. J. de Jong, and G. W. Somsen, "Developments in coupled solid-phase extraction-capillary electrophoresis 20092011," Electrophoresis, vol. 33, pp. 243-250, 2012.

[13] R. Barro, S. Ares, C. Garcia-Jares, M. Llompart, and R. Cela, "Development of a sensitive methodology for the analysis of chlorobenzenes in air by combination of solid-phase extraction and headspace solid-phase microextraction," Journal of Chromatography A, vol. 1045, no. 1-2, pp. 189-196, 2004.

[14] A. R. Khorrami and M. Edrisi, "Synthesis and evaluation of a molecularly imprinted polymer for solid phase extraction of ethopabate from chicken tissue," Separation Science and Technology, vol. 45, no. 3, pp. 404-412, 2010.

[15] J. Wolska and M. B. Jak, "Sorption of phthalates on molecularly imprinted polymers," Separation Science and Technology, vol. 47, pp. 1316-1321, 2012.

[16] R. Say, A. Ersöz, I. Şener, A. Atilir, S. Diltemiz, and A. Denizli, "Comparison of adsorption and selectivity characteristics for 4nitrophenol imprinted polymers prepared via bulk and suspension polymerization," Separation Science and Technology, vol. 39, no. 15, pp. 3471-3484, 2004.

[17] E. Turiel and A. Martín-Esteban, "Molecularly imprinted polymers for sample preparation: a review," Analytica Chimica Acta, vol. 668, no. 2, pp. 87-99, 2010.

[18] X. Jiang, N. Jiang, H. Zhang, and M. Liu, "Small organic molecular imprinted materials: their preparation and application," Analytical and Bioanalytical Chemistry, vol. 389, pp. 355-368, 2005. 
[19] J. P. Lai, M. L. Yang, R. Niessner, and D. Knopp, "Molecularly imprinted microspheres and nanospheres for di(2-ethylhexyl) phthalate prepared by precipitation polymerization," Analytical and Bioanalytical Chemistry, vol. 389, no. 2, pp. 405-412, 2007.

[20] H. Yan, X. Cheng, and G. Yang, "Dummy molecularly imprinted solid phase extraction for selective determination of five phthalate esters in plastic bottled functional beverages," Journal of Agricultural and Food Chemistry, vol. 60, pp. 5524-5531, 2012.

[21] P. Qi, J. Wang, Y. Li, F. Su, J. Jin, and J. Chen, "Molecularly imprinted solid phase extraction coupled with HPLC for the selective determination of monobutyl phthalate in bottled water," Journal of Separation Science, vol. 34, no. 19, pp. 27122718, 2011.

[22] J. He, R. Lv, H. Zhan et al., "Preparation and evaluation of molecularly imprinted solid-phase micro-extraction fibers for selective extraction of phthalates in an aqueous sample," Analytica Chimica Acta, vol. 674, no. 1, pp. 53-58, 2010.

[23] J. He, R. Lv, J. Zhu, and K. Lu, "Selective solid-phase extraction of dibutyl phthalate from soybean milk using molecular imprinted polymers," Analytica Chimica Acta, vol. 661, no. 2, pp. 215-221, 2010.

[24] H. Shaikh, N. Memon, H. Khan, M. I. Bhanger, and S. M. Nizamani, "Preparation and characterization of molecularly imprinted polymer for di(2-ethylhexyl) phthalate: application to sample clean-up prior to gas chromatographic determination," Journal of Chromatography A, vol. 1247, pp. 125-133, 2012.

[25] A. R. Khorrami and S. Mehrseresht, "Synthesis and evaluation of a selective molecularly imprinted polymer for the contraceptive drug levonorgestrel," Journal of Chromatography B, vol. 867, no. 2, pp. 264-269, 2008.

[26] K. Farrington, E. Magner, and F. Regan, "Predicting the performance of molecularly imprinted polymers: selective extraction of caffeine by molecularly imprinted solid phase extraction," Analytica Chimica Acta, vol. 566, no. 1, pp. 60-68, 2006.

[27] D. Spivak, M. A. Gilmore, and K. J. Shea, "Evaluation of binding and origins of specificity of 9-ethyladenine imprinted polymers," Journal of the American Chemical Society, vol. 119, no. 19, pp. 4388-4393, 1997.

[28] C. Crescenzi, S. Bayoudh, P. A. G. Cormack, T. Klein, and K. Ensing, "Determination of clenbuterol in bovine liver by combining matrix solid-phase dispersion and molecularly imprinted solid-phase extraction followed by liquid chromatography/ electrospray ion trap multiple-stage mass spectrometry," Analytical Chemistry, vol. 73, no. 10, pp. 2171-2177, 2001. 

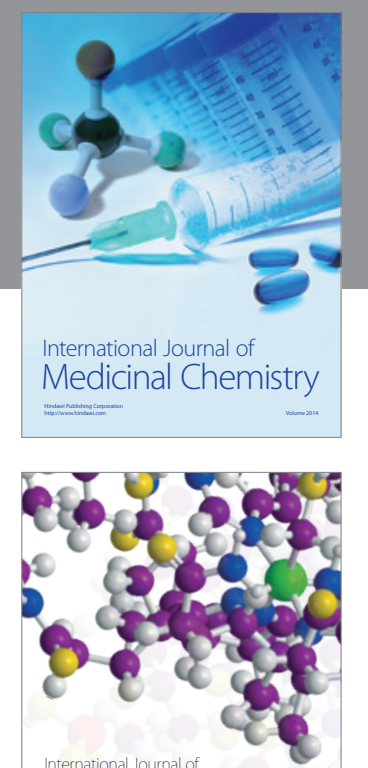

\section{Carbohydrate} Chemistry

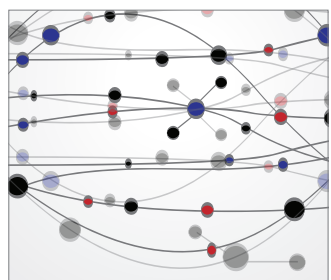

The Scientific World Journal
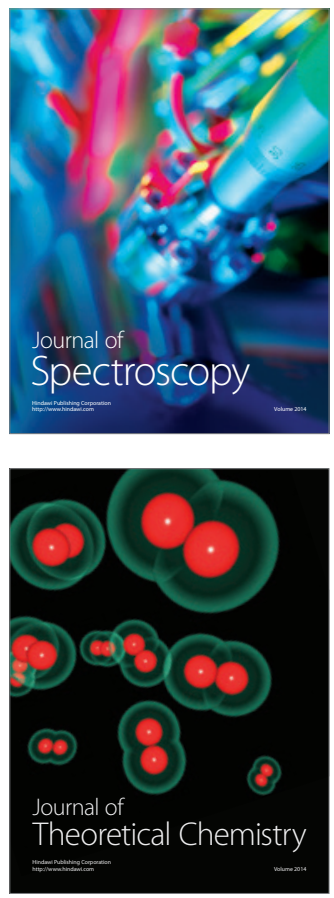
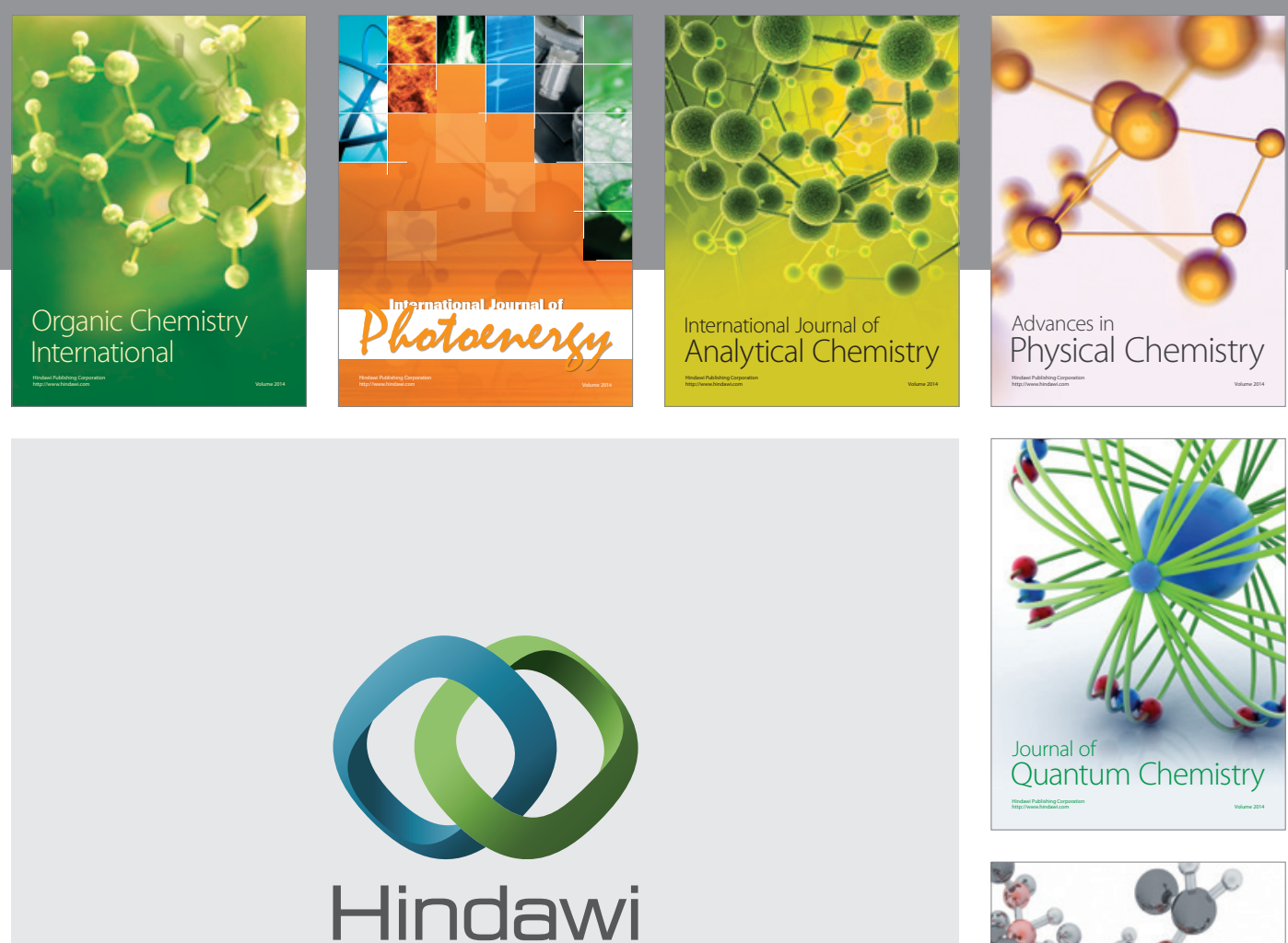

Submit your manuscripts at

http://www.hindawi.com

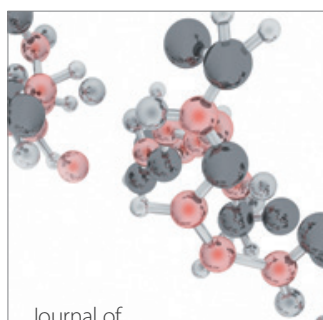

Analytical Methods

in Chemistry

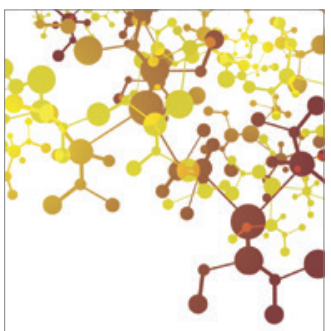

Journal of

Applied Chemistry

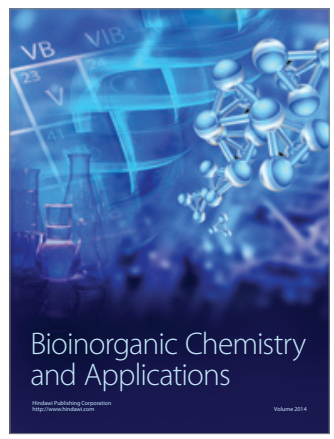

Inorganic Chemistry
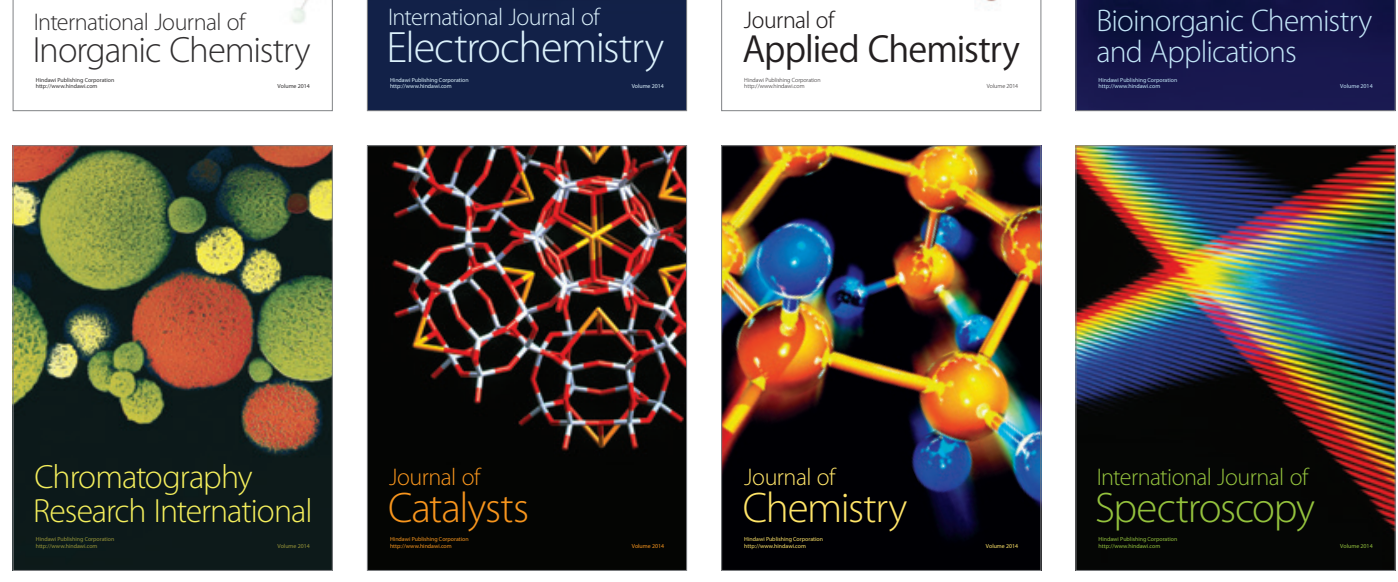